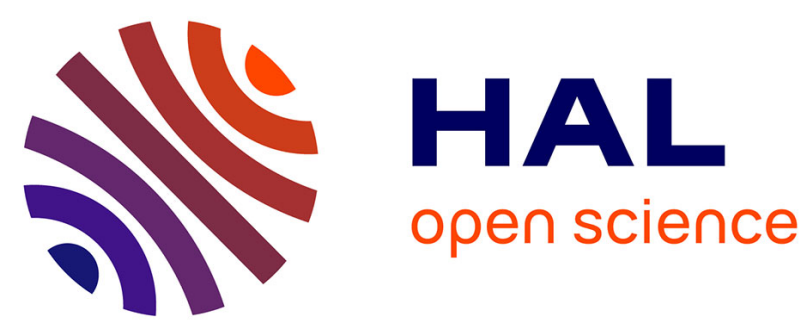

\title{
Calpains: Markers of tumor aggressiveness?
}

Hélène Roumes, Ludovic Leloup, Elise Dargelos, Jean-Jacques Brustis, Laetitia Daury, Patrick Cottin

\section{To cite this version:}

Hélène Roumes, Ludovic Leloup, Elise Dargelos, Jean-Jacques Brustis, Laetitia Daury, et al.. Calpains: Markers of tumor aggressiveness?. Experimental Cell Research, 2010, 316 (9), pp.1587 - 1599. 10.1016/j.yexcr.2010.02.017 . hal-01740972

\section{HAL Id: hal-01740972 https://hal-amu.archives-ouvertes.fr/hal-01740972}

Submitted on 31 May 2020

HAL is a multi-disciplinary open access archive for the deposit and dissemination of scientific research documents, whether they are published or not. The documents may come from teaching and research institutions in France or abroad, or from public or private research centers.
L'archive ouverte pluridisciplinaire HAL, est destinée au dépôt et à la diffusion de documents scientifiques de niveau recherche, publiés ou non, émanant des établissements d'enseignement et de recherche français ou étrangers, des laboratoires publics ou privés. 


\title{
Research Article
}

\section{Calpains: Markers of tumor aggressiveness?}

\author{
Hélène Roumes ${ }^{a}$, Ludovic Leloup ${ }^{b, 1}$, Elise Dargelos ${ }^{a}$, Jean-Jacques Brustis ${ }^{a}$, \\ Laetitia Daury $^{a, *}$, Patrick Cottin ${ }^{a}$ \\ aniversité Bordeaux 1, Unité Protéolyse, Croissance et Développement Musculaire, INRA USC-2009, Avenue des Facultés, \\ 33405 Talence Cedex, France \\ ${ }^{\mathrm{b}}$ Department of Pathology, S713 Scaife Hall, University of Pittsburgh, Pittsburgh, PA 15261, USA
}

\author{
Article Chronology: \\ Received 16 November 2009 \\ Revised version received \\ 5 February 2010 \\ Accepted 16 February 2010 \\ Available online 1 March 2010 \\ Keywords: \\ Calpains \\ Rhabdomyosarcoma cells \\ Migration \\ Invasion \\ Cytoskeleton
}

A R T I C L E I N F O R M A T I O N

\begin{abstract}
A B S T R A C T
Rhabdomyosarcoma (RMS) are soft-tissue sarcoma commonly encountered in childhood. RMS cells can acquire invasive behavior and form metastases. The metastatic dissemination implicates many proteases among which are $\mu$-calpain and m-calpain.

Study of calpain expression and activity underline the deregulation of calpain activity in RMS Analysis of kinetic characteristics of RMS cells, compared to human myoblasts LHCN-M2 cells, shows an important migration velocity in RMS cells. One of the major results of this study is the positive linear correlation between calpain activity and migration velocity presenting calpains as a marker of tumor aggressiveness. The RMS cytoskeleton is disorganized. Specifying the role of $\mu-$ and $\mathrm{m}$-calpain using antisense oligonucleotides led to show that both calpains up-regulate $\alpha$ - and $\beta$-actin in ARMS cells. Moreover, the invasive behavior of these cells is higher than that of LHCNM2 cells. However, it is similar to that of non-treated LHCN-M2 cells, when calpains are inhibited. In summary, calpains may be involved in the anarchic adhesion, migration and invasion of RMS. The direct relationship between calpain activity and migration velocities or invasive behavior indicates that calpains could be considered as markers of tumor aggressiveness and as potential targets for limiting development of RMS tumor as well as their metastatic behavior.
\end{abstract}

(c) 2010 Elsevier Inc. All rights reserved.
Alveolar and embryonal rhabdomyosarcoma (ARMS and ERMS respectively) are soft-tissue sarcoma commonly encountered in childhood and adolescence. These rhabdomyosarcoma (RMS) arise from immature cells which fate is to form striated skeletal muscle. These cells express, similarly to muscle cells, some muscle specific proteins such as myogenic factors (MRF), desmin, and myogenin $[1,2]$. ARMS are characterized by two chromosomal translocations: $\mathrm{t}(2 ; 13)(\mathrm{q} 35 ; \mathrm{q} 14)$ or $\mathrm{t}(1 ; 13)(\mathrm{p} 36 ; \mathrm{q} 14)$ [3]. ERMS histological type is associated with a loss of heterozygosis at the 11p15 locus [4]. RMS can acquire invasive behavior and can form metastases in lung, bone, marrow, and lymphatic nodes. The development of such metastases is associated with poor prognosis [5]. The comprehension of mechanisms that regulate cancer cells migration and invasion may be a key for the development of new therapies for limiting metastases. Among the many proteases implicated in cell motility, the calpains play an essential role in regulating migration and invasion phenomena, both involved in metastases development [6-8]. Targeting calpains may present a novel approach toward restraining metastasis and development of RMS cancers. Calpains are calcium-dependent cysteine proteases which best-characterized members are $\mu$-calpain and m-calpain (i.e. CAPN1 and CAPN2 respectively), known as ubiquitous

\footnotetext{
* Corresponding author. Tel.: +33 (0)5 400021 94; fax: +33 (0)5 40008496.

E-mail address: daury@enscbp.fr (L. Daury).

${ }^{1}$ Tel.: (412) 624 9366; fax: (412) 6248946.
} 
heterodimeric enzymes consisting of a large 80 -kDa catalytic subunit and a smaller regulatory subunit (about $28 \mathrm{kDa}$, css 1 or css2). Css1 is ubiquitous while css2 is tissue-specific [9].

Calpain activity is $\mathrm{Ca}^{2+}$-dependent. Among the different factors regulating calpain activity, the critical factor is its specific endogenous inhibitor, calpastatin [10]. Calpastatin is a ubiquitous protein regulated by several factors [11] that requires $\mathrm{Ca}^{2+}$ to bind and inhibits calpains [12].

The present study focuses on the role of both $\mu$ - and m-calpain in the metastatic characteristics of the two cancer cell lines, ARMS and ERMS, assuming the role of calpains in cell adhesion and migration (that are limited steps of invasion and metastasis).

To reach that goal, we first characterized the calpain system in RMS before studying the role of the calpains in RMS cell adhesion, on RMS morphology and cytoskeleton organization, as well as in RMS migration characteristics and invasive behavior. Non-tumor human myoblasts LHCN-M2 [13] were chosen as non-metastatic control cells.

The main result is that calpains play a crucial role in adhesion, high migration velocities and invasive behavior of RMS. More specifically, calpains may be considered as markers of tumor aggressiveness since their global activity is linked to migration velocities by a positive linear correlation. Moreover, inhibition of calpain activity in RMS led to migration velocities and invasive behavior similar to those of non-treated LHCN-M2 cells. Because calpain activity is directly involved in migration and invasion of RMS, the proteases would be a good target for the development of novel therapies to control metastasis.

\section{Materials and methods}

\section{Cell culture}

Cells were grown under $5 \% \mathrm{CO}_{2}$ atmosphere at $37^{\circ} \mathrm{C}$ in DMEM (Dulbecco's modified Eagle medium) with pyruvate supplemented with $10 \%$ FBS (fetal bovine serum) for RMS, and a culture medium containing DMEM without pyruvate (60\%), medium 199 (20\%) and FBS (20\%) for LHCN-M2 cells (human myoblasts cell lines) [13]. All cell culture reagents were purchased from Gibco-BRL. Some experiments have been done only with the tumor cell line presenting the most aggressive phenotype, ARMS.

\section{Calpain inhibitor treatments}

The chemical calpain inhibitor calpeptin (Z-Leu-Nle-CHO) was purchased from Calbiochem and used at 40 and $60 \mu \mathrm{M}$ for adhesion assay, $50 \mu \mathrm{M}$ for migration and invasion assay for immunolocalization and for cytoskeleton organization and $80 \mu \mathrm{M}$ for Western blot. A second calpain inhibitor, calpain inhibitor III (Z-Val-Phe-CHO, Calbiochem) was used at $50 \mu \mathrm{M}$. Antisense oligodeoxynucleotide transient transfections has been realized as previously described [14].

\section{Quantification of calpain activity}

All cell lines were cultured in DMEM supplemented with $0.1 \%$ FBS during $48 \mathrm{~h}$. The global calpain activity was observed and quantified on living cells using t-BOC-LM-CMAC (7-amino-4chloromethylcoumarin, $t$-BOC-L-leucyl-L-methionine amide,
Molecular Probes) [7,15]. This molecule enters passively in cells and becomes fluorescent after calpain cleavage. Culture medium was replaced by DMEM without serum containing $50 \mu \mathrm{M}$ of $t$-BOCLM-CMAC. Cells were incubated during $30 \mathrm{~min}$ in darkness. The $\mu-$ calpain activity was observed and quantified on fixed and permeabilized cells using Calpain 1 substrate (Chemicon) which become fluorescent after $\mu$-calpain cleavage. After PAF fixation (15 min) and permeabilization with $1 \%$ Triton X-100 (3 min), cells were incubated with Calpain 1 substrate $50 \mu \mathrm{M}$ during $40 \mathrm{~min}$ in darkness. The global calpain or the $\mu$-calpain activities were then observed using an inverted epifluorescence microscope (Leica AF DMI6000). The results were quantified using the Metamorph software (Molecular Device).

\section{Immunolocalization of calpains and calpastatin}

All cell lines were cultured in DMEM supplemented with $0.1 \%$ FBS for $48 \mathrm{~h}$. Next, cells were fixed and permeabilized. A specificity was disrupted by incubation ( $1 \mathrm{~h}$ ) with PBS/BSA (3\%). Then, m-calpain, $\mu$-calpain and calpastatin were detected using corresponding antibodies (N-19 sc-7533 raised in goat (1/50), Santa Cruz Biotechnology; MAB3104, raised in mouse (1/500), Chemicon; H-300 sc-20779, raised in rabbit (1/50), Santa Cruz Biotechnology respectively, for $3 \mathrm{~h}$ ). Then, cells were incubated with an appropriate secondary Alexa fluor antibody (anti-goat-Alexa594conjugated (1/1000), anti-mouse-Alexa594-conjugated (1/3000), and anti-rabbit-Alexa488-conjugated $(1 / 1000)$ antibodies respectively for $90 \mathrm{~min}$ ). Next cells were observed using an epifluorescence microscope (Leica AF DMI6000).

\section{Adhesion assay}

Adhesion assays were performed as described previously [14]. These assays were carried out with $(40 \mu \mathrm{M}$ or $60 \mu \mathrm{M})$ or without calpeptin treatment.

\section{Migration assay}

All cell lines were seeded in DMEM containing $0.1 \%$ FBS at a density of $4.10^{3}$ cells $/ \mathrm{cm}^{2}$ in $35 \mathrm{~mm}$ diameter glass-bottom plate. Eight hours after seeding, time-lapse microscopy experiments were performed on an inverted Leica AF DMI6000 microscope equipped with an environmental chamber with phase-contrast optic (images taken every $15 \mathrm{~min}$ ). Cell velocities of migration, defined as the average of 73 subsequent cell centroid displacements/one time interval between two successive images (15 $\mathrm{min}$ ), were evaluated using the tracking object of the Metamorph software (Molecular Device). The cell trajectories were recorded for $18 \mathrm{~h}$. These assays have been made in presence vs. absence of calpeptin $(50 \mu \mathrm{M})$ added at the beginning of the time-lapse.

\section{Invasion assay}

The invasive capacity of RMS and LHCN-M2 cells was measured using BD Biocoat Growth Factor Reduced Matrigel Invasion Chambers (BD Biosciences). Cells were seeded at a density of $10^{5}$ cells $/ \mathrm{cm}^{2}$ on the membrane in DMEM with $0.1 \% \mathrm{FBS}$. Quiescent medium was also added under the membrane. After $48 \mathrm{~h}$ of incubation, cells staying on the membrane were scraped off. Cells lying under the membrane were fixed with PAF (4\%), and 
dehydrated overnight using ethanol. Cells were then stained with Hansen's hemalun and evaluated. The same experiment was realized with culture insert whose PET membrane was recovered by BD Matrigel matrix. Cells crossing the membrane were stained and evaluated. The invasive capacity of all cell lines was expressed by the percentage of cells going through the membrane with Matrigel matrix as compared to the percentage of cells going through the membrane without any Matrigel matrix. The implication of calpains in this phenomenon was evaluated using the same procedures after calpeptin treatment $(50 \mu \mathrm{M})$.

\section{Cytoskeleton analysis and vinculin localization}

Cell actin fibers were stained as previously described [16], with vs. without calpeptin or inhibitor III, and observed using an epifluorescence microscope (Leica AF DMI6000). Vinculin was detected after fixation and permeabilization, using primary antibody (V-9131, Sigma-Aldrich; 1/50) (3 h). Next, cells were incubated with a secondary Alexa fluor antibody from Molecular Probes (anti-mouse-Alexa594-conjugated 1/1000) (90 min).

\section{Western blots}

Western blots were performed as described previously [16]. The bicinchoninic acid protein assay kit was purchased from Pierce, polyvinylidene fluoride membrane from Millipore and nitroblue tetrazolium/5-bromo-4chloro-3-indoyl-1-phosphate from Promega. The membranes were incubated with specific antibodies against $\mathrm{m}$-calpain (raised in sheep as previously described ([17]; 1/1000), $\beta$-tubulin (Santa Cruz Biotechnology (H-235 sc9104); 1/500), $\mu$-calpain (Sigma-Aldrich (C-0355); 1/2500) and calpastatin (Santa Cruz Biotechnology (H-300 sc-20779); $1 / 250$ ). The secondary antibody conjugated to alkaline phosphatase (Sigma-Aldrich; 1/10,000) was used for detection. The apparent density of the band on the membranes was quantified with Totalab (Progenesis) and corrected according to $\beta$-tubulin expression.

\section{Extraction of total RNA, reverse transcription and quantitative PCR}

Extraction of total RNA, reverse transcription and quantitative PCR were performed as previously described [18]. Forward and reverse primer sequences for $\mu$ - and m-calpain amplification were agtcgtgccgcagcatggtga, acttgtccaggtcaaacttccg and gccatcaagtacctcaaccagg, gctccttggcagatgtctgtg, respectively. Transcript concentration of both isoforms was determined from the standard curve and compared to the control LHCN-M2. GAPDH (glyceraldehyde phosphate dehydrogenase) was used as control; its forward and reverse primer sequences were aaggtcatccagagctgaacgc and acaacctggtcctcagtgtagcc.

\section{Statistical analysis}

The statistical significance of the differences between multiple groups was determined using ANOVA. When $F$ was associated with a probability $p<0.05$, intergroup comparisons were conducted using Student's t-test. Unless indicated, all data were expressed as means \pm SEM and were representative of an average of at least three independent experiments.

\section{Results}

\section{Characterization of the calpain system in rhabdomyosarcoma cells}

In RMS cells, the expression of $\mu$-calpain and m-calpain was significantly reduced compared to that of LHCN-M2 cells (Fig. 1A1-2). Moreover, the expression of calpastatin was reduced by $85 \pm 1 \%$ in ARMS cells and $74 \pm 3 \%$ in ERMS cells, compared to that of LHCN-M2 cells (Fig. 1A3).

Specific mRNA levels for $\mu$ - and m-calpains were quantified by real time RT-PCR and compared to that of LHCN-M2 control cells (Fig. 1B). The mRNA level of $\mu$-calpain was similar in all cell lines (Fig. 1B1) whereas the expression level of m-calpain mRNA was reduced by more than 40\% and 50\% for ARMS and ERMS cells, respectively (Fig. 1B2).

Using $t$-BOC-LM-CMAC, the global calpain activity of RMS cells was measured and compared to that of LHCN-M2 cells (Fig. 1C1-3). Calpain activity was significantly higher in both RMS cell lines than in LHCN-M2 cells $(2.6 \pm 0.6$ and $2.1 \pm 0.1$ fold for ARMS and ERMS cells respectively) (Fig. 1C4). Furthermore, $\mu$-calpain activity (calpain 1 substrate) was higher in RMS than in LHCN-M2 cells $(2.3 \pm 0.4$ and $2.7 \pm 0.4$ fold for ARMS and ERMS cells respectively) (Fig. 1C5).

In LHCN-M2 myoblasts, $\mu$-calpain was concentrated around the nucleus with a weak decreasing gradient towards the cell periphery presenting a linear structure parallel to the main axis of the cell (Fig. 1D1). In these cells, m-calpain was more particularly localized around the nucleus in a spot-shaped fashion (Fig. 1D2) and calpastatin presented a higher concentration around the nucleus, decreasing towards the cell periphery (Fig. 1D3). In RMS cells, $\mu$-calpain presented a higher concentration around the nucleus, decreasing towards the cell periphery. mCalpain and calpastatin localizations were not different between all cell lines (Fig. 1D4-6 for ARMS, data not shown for ERMS cells).

\section{Implication of calpains in adhesion}

As shown in Fig. 2A, RMS cells presented a delay and a decrease in their adhesion rates. Eight hours after seeding, the adhesion rates of ARMS and ERMS were $37 \pm 3 \%$ and $35 \pm 4 \%$ respectively, whereas LHCN-M2 myoblasts adhered completely $(p<0.05)$.

Calpain inhibition drastically decreased the adhesion rate of LHCN-M2 (Fig. 2B1). The presence of calpeptin led to a decrease in the adhesion rate of ARMS. However, this decrease was significant only 2 and $4 \mathrm{~h}$ after seeding at $40 \mu \mathrm{M}$ of calpeptin and after $2 \mathrm{~h}$ at $60 \mu \mathrm{M}$ of calpeptin (Fig. 2B2). The adhesion rates of ERMS were not affected by the presence of calpeptin neither at $40 \mu \mathrm{M}$ of calpeptin nor at $60 \mu \mathrm{M}$ (data not shown).

In order to understand the difference of adhesiveness between RMS and LHCN-M2 cells, vinculin (one of the main focal adhesion components) localization was investigated in all cell lines (Fig. 2). In LHCN-M2 cells, vinculin was regularly localized at the ventral surface of the cell (Fig. 2C1). On the contrary, in both RMS cell lines, vinculin was principally localized at the periphery of the cell (Fig. 2C2 for ARMS, data not shown for ERMS). The inhibition of calpain activity led to a reorganization of vinculin. In all cell lines presenting a rounded shape, vinculin was localized predominantly at the periphery of the ventral surface of the cell (Fig. 2C3 and C4 

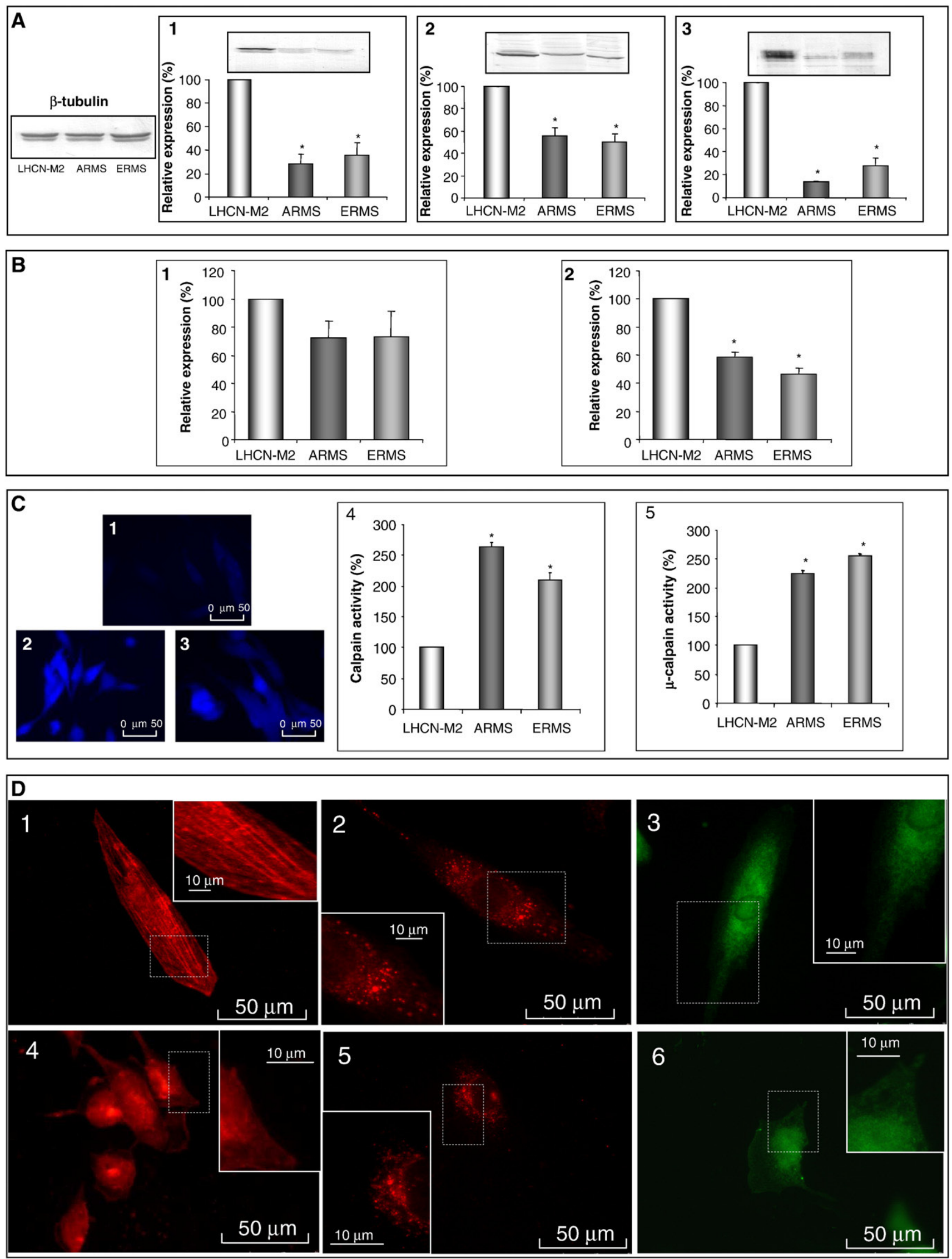
for LHCN-M2 and ARMS cells, respectively, data not shown for ERMS). Contrary to LHCN-M2 myoblasts, in which the presence of calpeptin led to a $107 \pm 14 \%$ increase in vinculin expression, the presence of calpeptin in ARMS led to a $14 \pm 3 \%$ decrease in vinculin expression (Fig. 2C5). Antisense oligodeoxynucleotide treatments against $\mu$-calpain led to an increase in LHCN-M2 vinculin expression $(14 \pm 4 \%)$ but did not affect the vinculin expression levels of ARMS ( $p=0.30$ ) (Fig. 2C6-7). Antisense oligodeoxynucleotide treatments against $\mathrm{m}$-calpain led to an increase in vinculin expression in LHCN-M2 cells (Fig. 2C6). Conversely, this treatment in ARMS led to a decrease in vinculin expression (Fig. 2C7).

\section{Morphological characteristics and actin cytoskeleton organization of migrating rhabdomyosarcoma cells}

Eight hours after seeding, control cells exhibited an elongated shape and membrane protrusions. Migrating RMS cell lines exhibited an elongated shape with a reduced cytoplasm and large nuclei. In addition, membrane protrusions were smaller and thinner than those of LHCN-M2 myoblasts (Fig. 3A1 and 3A2 for LHCN-M2 and ARMS cells respectively; data not shown for ERMS). The inhibition of calpain activity impaired cell morphology, all cell lines presenting a rounded shape with very short membrane protrusions (Fig. 3B1 and B2 for LHCN-M2 and ARMS cells, respectively; data not shown for ERMS).

Since the actin cytoskeleton is responsible for cell morphology and considered to be the motor of cell migration [19], we studied its organization in the different cell lines. In elongated LHCN-M2 cells, the actin cytoskeleton formed stress fibers parallel to the main axis of the cell (Fig. 3C1). On the contrary, in both RMS cell lines, actin cytoskeleton failed to form parallel stress fibers (Fig. 3C2 for ARMS; data not shown for ERMS). The actin cytoskeleton was disorganized and mainly concentrated at the periphery of the cell.

In presence of calpeptin, the LHCN-M2 F-actin was no longer at the center of the cell. However it still remained organized at the periphery of the cell where it was concentrated (Fig. 3C3). In both RMS cell lines, the inhibition of calpains induced an increase in the concentration of F-actin at the periphery of the cell (Fig. 3C4, data not shown for ERMS).

In all cell lines, the presence of calpeptin led to an increase in the quantity of F-actin (Fig. 3D1). This result was confirmed by the use of calpain inhibitor III leading to an increase in the level of Factin by $18 \pm 3 \%$ in ARMS (Fig. 3D2).

\section{Implication of calpain in the arrangement of actin cytoskeleton}

In LHCN-M2 cells, F-actin and $\mu$-calpain colocalized by $93 \%$ (F-actin overlapping $\mu$-calpain: $93.2 \pm 0.5 \%$ and $\mu$-calpain overlapping $\mathrm{F}$ actin: $93.2 \pm 1.0 \%$ ) (Fig. 4A1). In these cells, stress fibers were associated with a peak of $\mu$-calpain concentration (Fig. 4A2). In addition, F-actin in ARMS cells colocalized with $\mu$-calpain at a lower extent than in LHCN-M2 myoblasts (F-actin overlapping $\mu$ calpain: $66.5 \pm 3.5 \%$ and $\mu$-calpain overlapping F-actin: $46.7 \pm 2.9$ ) (Fig. 4A3-4). In both cell lines, the presence of calpeptin led to a relocalization of F-actin as well as $\mu$-calpain at the periphery of the cell (Fig. 4A5-8).

In order to precise the role of each calpain on $\alpha$-actin and $\beta$ actin expression (components of F-actin), the expression of each isoform of actin monomer was determined in the presence vs. absence of calpeptin. In LHCN-M2 cells, the inhibition of calpains induced an increase of both $\alpha$-actin and $\beta$-actin ( $\alpha$-actin increased by $51 \pm 14 \%$ and $\beta$-actin increased by $33 \pm 7$ ) (Fig. 4B1-2). In ARMS conversely, this inhibition induced a decrease of both $\alpha$ actin and $\beta$-actin ( $\alpha$-actin decreased by $38 \pm 5 \%$ and $\beta$-actin decreased by $15 \pm 3 \%$ ) (Fig. 4B1-2).

Moreover, in LHCN-M2 cells, the inhibition of $\mu$-calpain (by antisense oligodeoxynucleotide treatments) up-regulated the expression of $\alpha$-actin but did not affect the expression level of $\beta$-actin (Fig. 4B3 and 5) while the inhibition of m-calpain downregulated the expression of $\alpha$-actin and up-regulated the expression of $\beta$-actin (Fig. 4B4 and 6). It appeared that the inhibition of $\mu$ calpain as well as m-calpain in ARMS down-regulated the expression of both $\alpha$-actin and $\beta$-actin (Fig. 4B4 and 6).

\section{Kinetic characteristics of rhabdomyosarcoma cell lines}

As presented in Fig. 5A, the migration velocities of cancer cells were significantly higher (about 2.3 and 1.6 fold for ARMS and ERMS respectively) than those measured in controls. The inhibition of calpain activity drastically impaired migration velocities of all cell lines (Fig. 5A). The velocities decreased by $11 \%$ for LHCNM2, 60\% for ARMS and 28\% for ERMS cells. Similar results were obtained using the calpain inhibitor III (data not shown).

To complete this study, the different cell trajectories were analyzed and are presented in Fig. 5B. The ARMS dispersion area was higher than that of LHCN-M2 cells (Fig. 5B2 vs. B1, ERMS dispersion area was intermediate, data not shown). As presented in Fig. $5 \mathrm{C} 1, \Delta$ (vector between the initial point of each trajectory and the final localization of the cell) confirmed that the dispersive capacity was higher for the ARMS cell line. The inhibition of calpain activity significantly impaired the dispersion area of all cell lines (Fig. 5B3 vs. B1 and B4 vs. B2) as well as the dispersive behavior (Fig. 5C2-3; data not shown for ERMS). The dispersive capacity decreased by $35 \%, 44 \%$ and $49 \%$ for LHCN-M2, ARMS and ERMS cells respectively. Moreover, the dispersive capacity of treated ARMS cells was similar to the dispersive capacity of LHCN-M2 in standard conditions $(p=0.07)$.

These results highlight the role of calpains in migration and dispersive behavior of all cell lines. Moreover, the global activity of calpains was directly correlated with the velocity of each cell

Fig. 1 - Characterization of calpain system components in rhabdomyosarcoma cells. Expression of $\mu$-calpain, $\mathrm{m}$-calpain and calpastatin in all cell lines (A1, A2, and A3, respectively). Quantification of $\mu$-calpain and $\mathrm{m}$-calpain mRNA expression, in all cell lines (B1 and B2, respectively). Global calpain activity in LHCN-M2, ARMS and ERMS cells (C1, C2, and C3, respectively). Quantification of global calpain activity and $\mu$-calpain activity (C4 and C5, respectively). Localization of $\mu$-calpain, $\mathrm{m}$-calpain and calpastatin in LHCN-M2 cells (D1, D2, and D3, respectively) and in ARMS cells (D4, D5, and D6, respectively). Results are mean values \pm SEM from at least 3 independent experiments. *Significantly different from the control LHCN-M2 cells $(p<0.05)$. Pictures shown are representative of at least three separated sets of culture. 

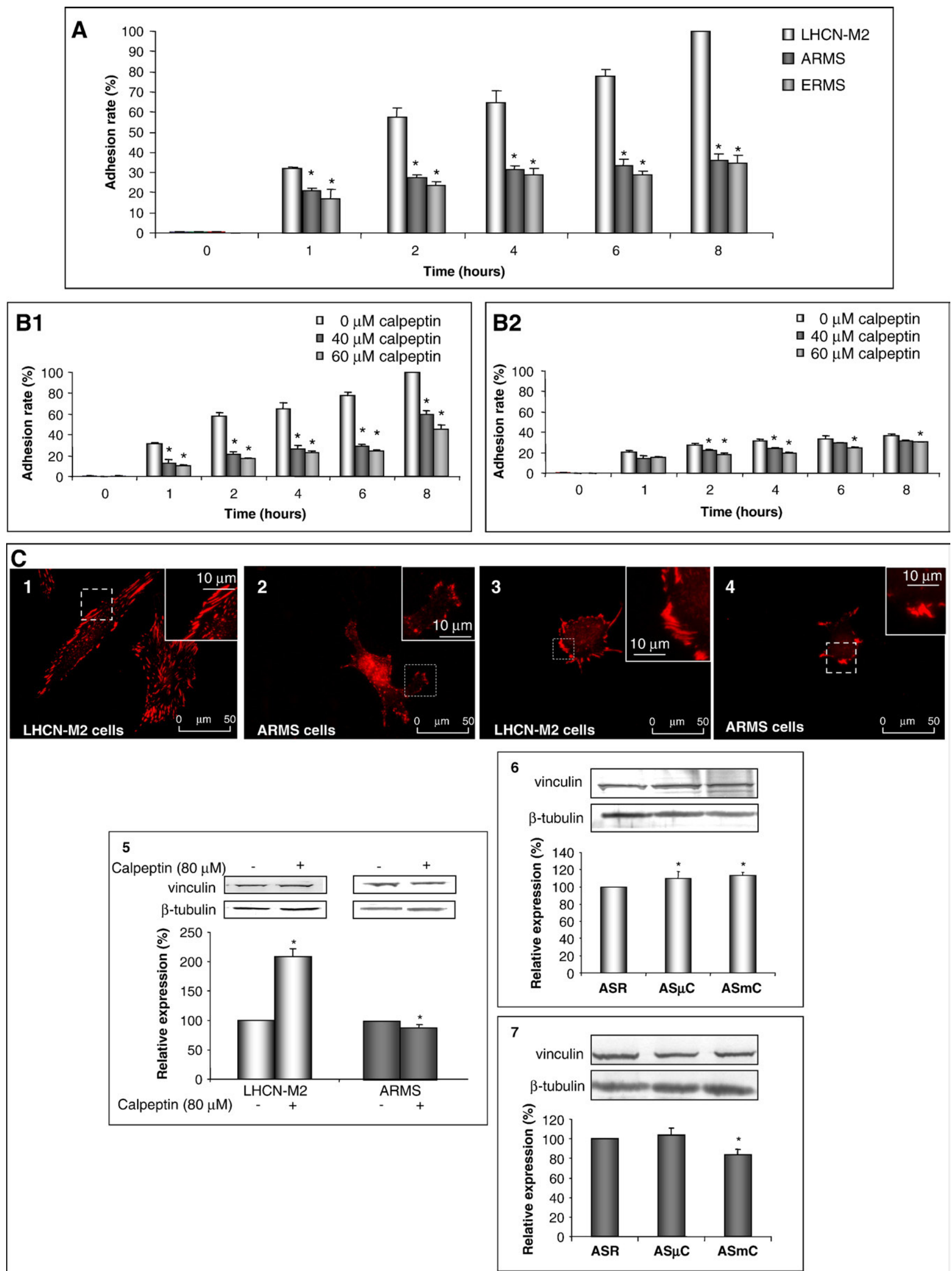

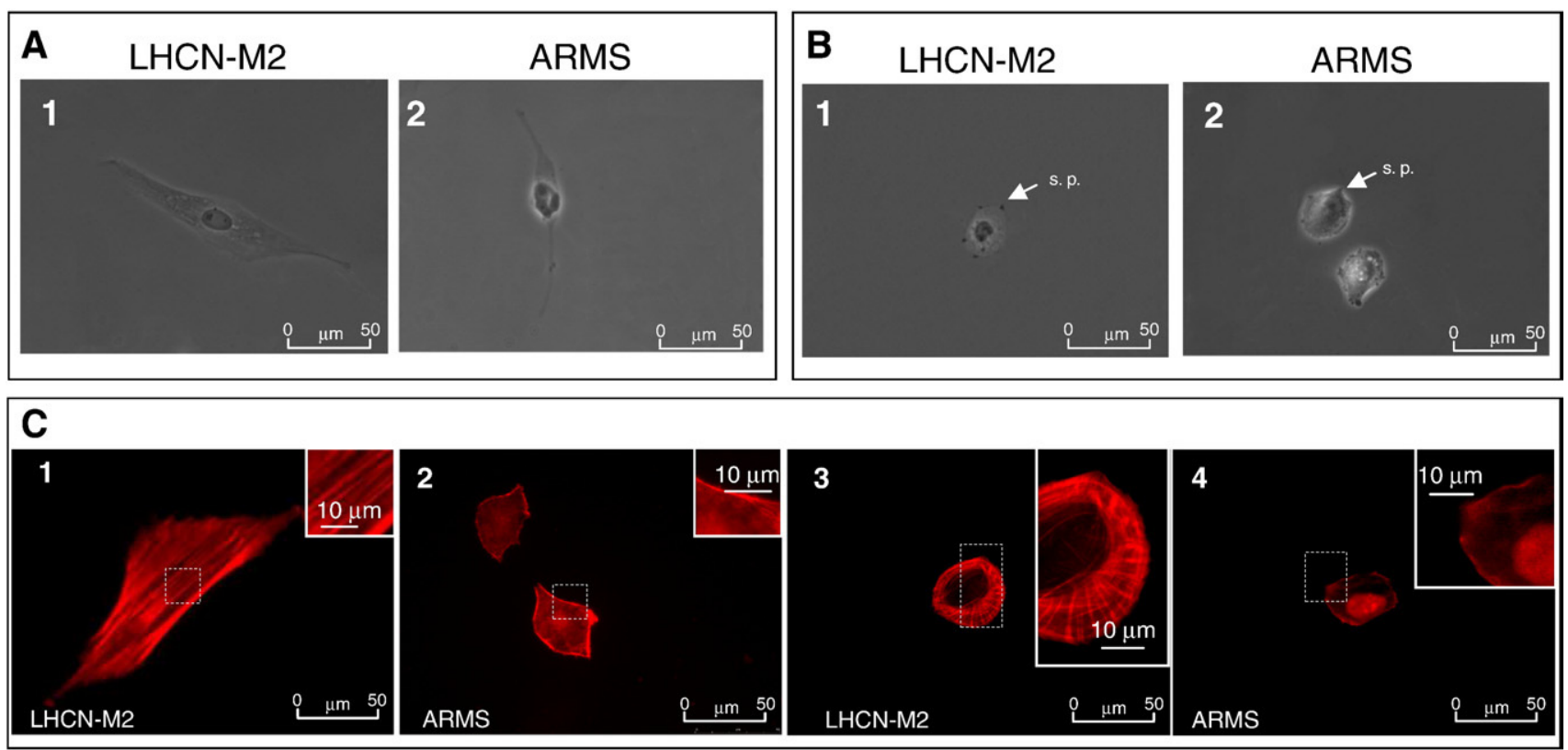

D
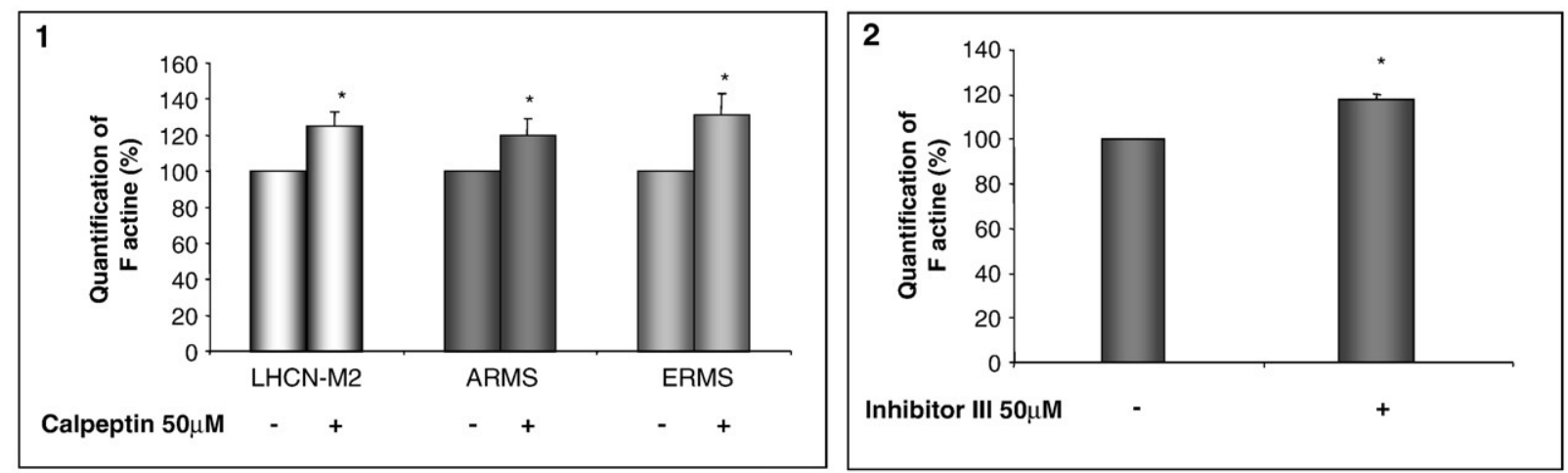

Fig. 3 - Morphological characteristics and actin cytoskeleton organization of migrating rhabdomyosarcoma cells. LHCN-M2 and ARMS cell morphology in standard conditions (A1 and A2, respectively) and in the presence of calpeptin (50 $\mu \mathrm{M})$ (B1 and B2, respectively). Cytoskeleton organization of LHCN-M2 and ARMS, in standard condition (C1 and C2, respectively) and in the presence of calpeptin ( $50 \mu \mathrm{M})$ (C3 and C4, respectively). Quantification of F-actin quantity, in all cell lines, with or without calpeptin treatment (50 $\mu \mathrm{M})$ (D1). Quantification of ARMS F-actin with or without calpain inhibitor III treatment (D2). Results are mean values \pm SEM from at least 3 independent experiments. Pictures shown are representative of at least three separated sets of culture. *Significantly different from the control $(\boldsymbol{p}<0.05)$.

line (Fig. 5D). Indeed, calpain activity and migration velocity showed a strong positive linear correlation: $y=13.71 x-0.395$ $\left(R^{2}=0.9401\right)$.

\section{Invasive behavior of rhabdomyosarcoma cells}

All cell lines were capable of breaching the Matrigel. Nevertheless, the invasive capacities of both RMS were significantly higher than that of control cells (Fig. 6A). The invasive index was $11.23 \pm 0.45$ for ARMS and $12.32 \pm 0.20$ for ERMS cell lines (Fig. 6B).

The inhibition of calpain activity led to a significant decrease in the invasive behavior of all cell lines. The invasive behavior of LHCN-M2 myoblasts decreased by about 2 fold. In the case of RMS cell lines, it decreased by more than 10 fold. Consequently, the invasive behavior of RMS treated with calpeptin as well as their invasive factor were equivalent to that of non-treated LHCN-M2

Fig. 2 - Implication of calpains in cell adhesion. Adhesion rates of all cell lines (A) and adhesion rates of LHCN-M2 (B1) and ARMS cells (B2) in calpeptin presence $(40 \mu \mathrm{M}$ or $60 \mu \mathrm{M})$ or not. Localization of vinculin in LHCN-M2 and ARMS cells (C1 and C2, respectively) or in LHCN-M2 and ARMS treated with calpeptin (50 $\mu \mathrm{M})$ (C3 and C4), respectively. Expression of vinculin in LHCN-M2 and ARMS cells treated or not with calpeptin ( $80 \mu \mathrm{M})(\mathrm{C5})$. Expression of vinculin in LHCN-M2 and ARMS (C6 and C7, respectively) transfected with antisense oligodeoxynucleotides directed against $\mu$-calpain or $\mathbf{m}$-calpain. Results are mean values $\pm S E M$ from at least 3 independent experiments. *Significantly different from the control (LHCN-M2 cells (A), non-treated cells (B1, B2, and C5) or cells transfected with random $(C 6, C 7))(p<0.05)$. Pictures shown are representative of at least three separated sets of culture. 

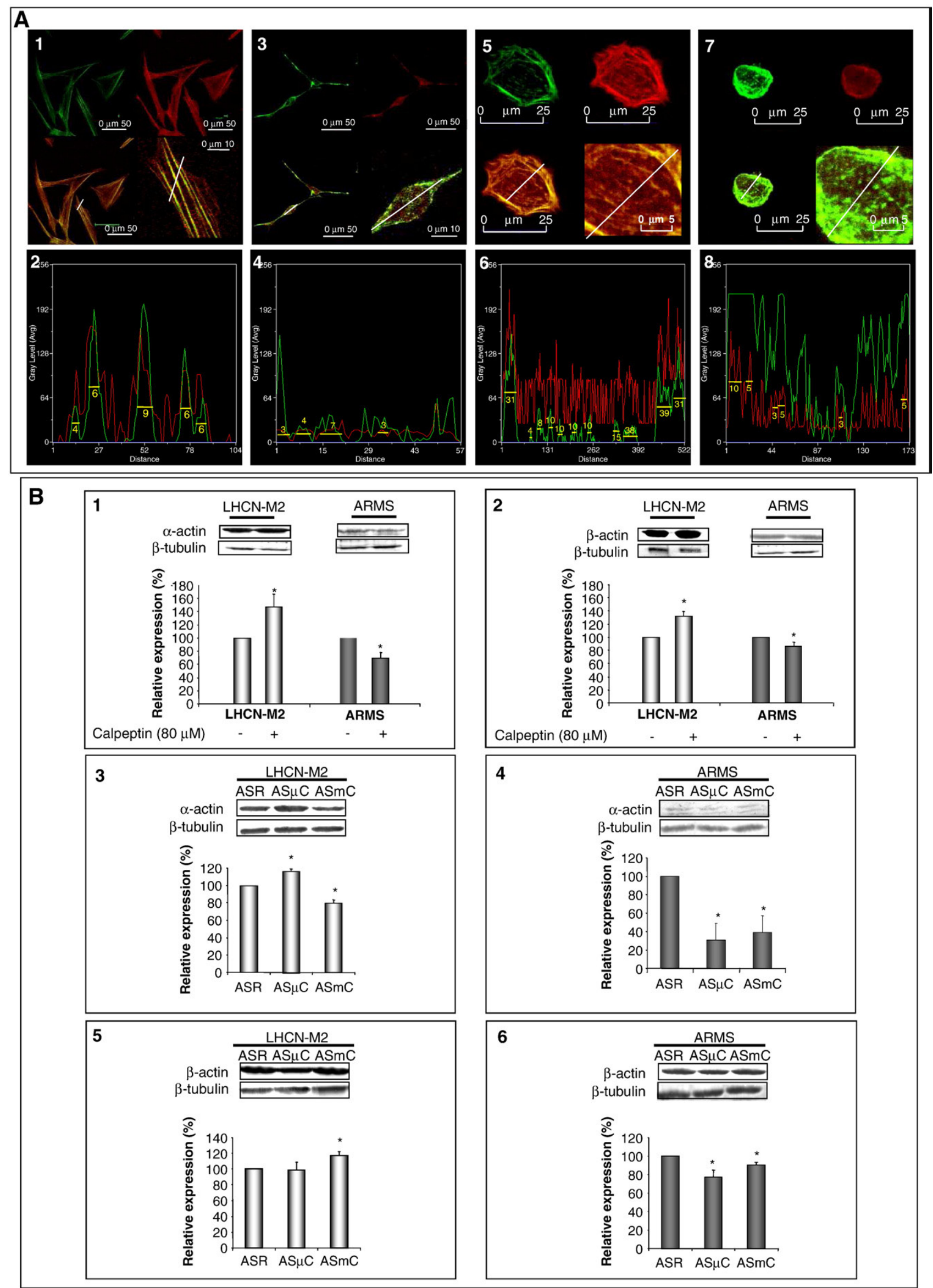
cells (Fig. 6A-B). Similar results were obtained using the calpain inhibitor III (data not shown).

\section{Discussion}

RMS cells can acquire invasive behavior and can form metastases in lung, bone, marrow, and lymphatic nodes decreasing the chances of patients' healing to less than $20 \%$ [5]. The comprehension of the mechanisms that regulate cancer cells migration and invasion may be a key for the development of new therapies for limiting metastases.

The determination of the mRNA expression level of $\mu$-calpain and $\mathrm{m}$-calpain, in all cell lines showed that it was similar for $\mu-$ calpain and it was reduced for m-calpain, in RMS cells, compared to control. However, Western blots showed that $\mu$-calpain as well as m-calpain were reduced in RMS when compared to control. These results could be explained by a potential post-transcriptional regulation for RMS $\mu$-calpain and by an absence of such regulation for m-calpain. In RMS cell lines, even though the expression of the calpain system components was decreased compared to LHCN-M2 myoblasts, both global and $\mu$-calpain activities were higher than in LHCN-M2 cells. This deregulation of RMS calpain system is in agreement with the high activity or expression of calpains detected in human renal cell carcinoma [20], in squamous cell carcinoma [21], and in human colorectal adenocarcinoma [22]. In RMS cells, calpastatin expression is highly down-regulated. Thereby, the increase in calpain activity could be due to an accumulation of the non-calpastatin-inhibited proteolyzed active forms of the proteases. The immunolocalization of calpain system components was different between the cell lines. In RMS cells, $\mu$-calpain presented a gradient of concentration decreasing toward the periphery of the cell. Conversely, in LHCN-M2 cells, $\mu$-calpain presented linear structures parallel to the main axis of the cell. This difference of $\mu$-calpain organization between LHCN-M2 and RMS cells presumes a different role for this protease as a function of the cell type. The localization of m-calpain was identical in all cell types and this observation confirms the results obtained by Samanta et al. showing the localization of $\mathrm{m}$ calpain at the endoplasmic reticulum level [23].

In RMS cells, the weak adhesion rate compared to LHCN-M2 is characteristic of the metastatic phenotype of these cells. In both RMS cell lines, the quantity of focal adhesions, marked by vinculin, was weaker than that of LHCN-M2 cells. Moreover, in RMS, vinculin was concentrated essentially at the periphery of the cell.
This vinculin distribution may be responsible for the weak adhesion rate of RMS. These results are in agreement with the decreased adhesiveness observed in circulating tumor cells in prostate cancer [24]. A reduction in the adhesiveness may increase the motility of RMS cells which is a key step of invasion [25]. Inhibition of calpain activity impaired the adhesion of LHCN-M2 and ARMS cells involving calpains in this process. Moreover, the inhibition of $\mu$-calpain as well as m-calpain led to an overexpression of vinculin in LHCN-M2 cells. These results indicate that vinculin is a calpain substrate in human myoblasts, in agreement with the results obtained by Taylor et al. in Z-disc [26]. In ARMS, the expression of vinculin was regulated only by m-calpain that stimulated its expression. Together, these results show the role of calpain in adhesion of LHCN-M2 and ARMS. They also support a differential role for $\mu$-calpain and $m$-calpain in the regulation of vinculin depending on the cell type. These differences at the adhesive capacity level may be responsible for some differences at the migration level.

Indeed, in all cell lines, the determination of migration velocities showed that RMS migrated faster and further away than LHCN-M2. The high migration velocities of RMS cells linked to the high invasive behavior of these cell lines are both characteristics of tumor and metastatic cells. In LHCN-M2 as well as in RMS cell lines, the inhibition of calpain activity led to a decrease in cell migration velocities. This decrease is in agreement with the decrease of migration observed in fibroblasts capn $4^{-/-}$[27]. Moreover, in presence of calpeptin, the migration velocities of ARMS were similar to the migration velocities of LHCN-M2 cells in standard conditions $(p=0.52$, NS). Similarly, the dispersion behavior of all cell lines was reduced and in presence of calpeptin, the dispersive capacity of ARMS was similar to the dispersive capacity of LHCN-M2 cells in standard conditions. Together, these results indicate that calpains are involved in migration velocities and dispersive capacity of migrating cells.

To summarize, RMS showed a high calpain activity and high migration velocities. One of the main results was the determination of a positive linear correlation between migration velocities and calpain activity. Indeed, calpain activity was directly linked to migration velocities pointing these proteases as markers of tumor aggressiveness.

The reorganization of the actin cytoskeleton is a fundamental step in cell adhesion, migration and invasion [19]. In LHCN-M2 cells, the actin cytoskeleton was organized in stress fibers that were parallel to the main axis of the cell. This organization confers a strong adhesiveness to these cells. Moreover, the F-actin

Fig. 4 - Implication of calpain in the actin cytoskeleton arrangement. Immunolocalization of F-actin (green) and $\mu$-calpain (red) in LHCN-M2 cells, in standard conditions (A1). Representation of colocalization of F-actin (green) and $\mu$-calpain (red), at the level of the line drawn on A1 (A2). A width at the middle of the peak overlapping zone higher than 2 pixels illustrated colocalization of both F-actin and $\mu$-calpain (white line). Numbers indicate the amount of pixel between the limit of the overlapping zone (A2). Immunolocalization and representation of colocalization of F-actin and $\mu$-calpain in ARMS cells, in standard conditions (A3 and A4, respectively). Immunolocalization and representation of colocalization of F-actin and $\mu$-calpain in LHCN-M2 and in ARMS, in the presence of calpeptin ( $50 \mu \mathrm{M})$ (A5 and A6, respectively, for LHCN-M2 cells and A7 and A8, respectively, for ARMS cells). Quantification of $\alpha$-actin and $\beta$-actin expression in LHCN-M2 and ARMS cells with or without calpeptin (80 $\mu$ M) (B1 and B2, respectively). Quantification of $\alpha$-actin expression in LHCN-M2 and ARMS cells after antisense oligonucleotides treatment (B3 and B4, respectively) and quantification of $\beta$-actin expression in LHCN-M2 and ARMS cells, in the same conditions (B5 and B6). Results are mean values \pm SEM from at least 3 independent experiments. *Significantly different from the control (non-treated cells or cells transfected with random) $(\boldsymbol{p}<\mathbf{0 . 0 5})$. Pictures shown are representative of at least three separated sets of culture. 

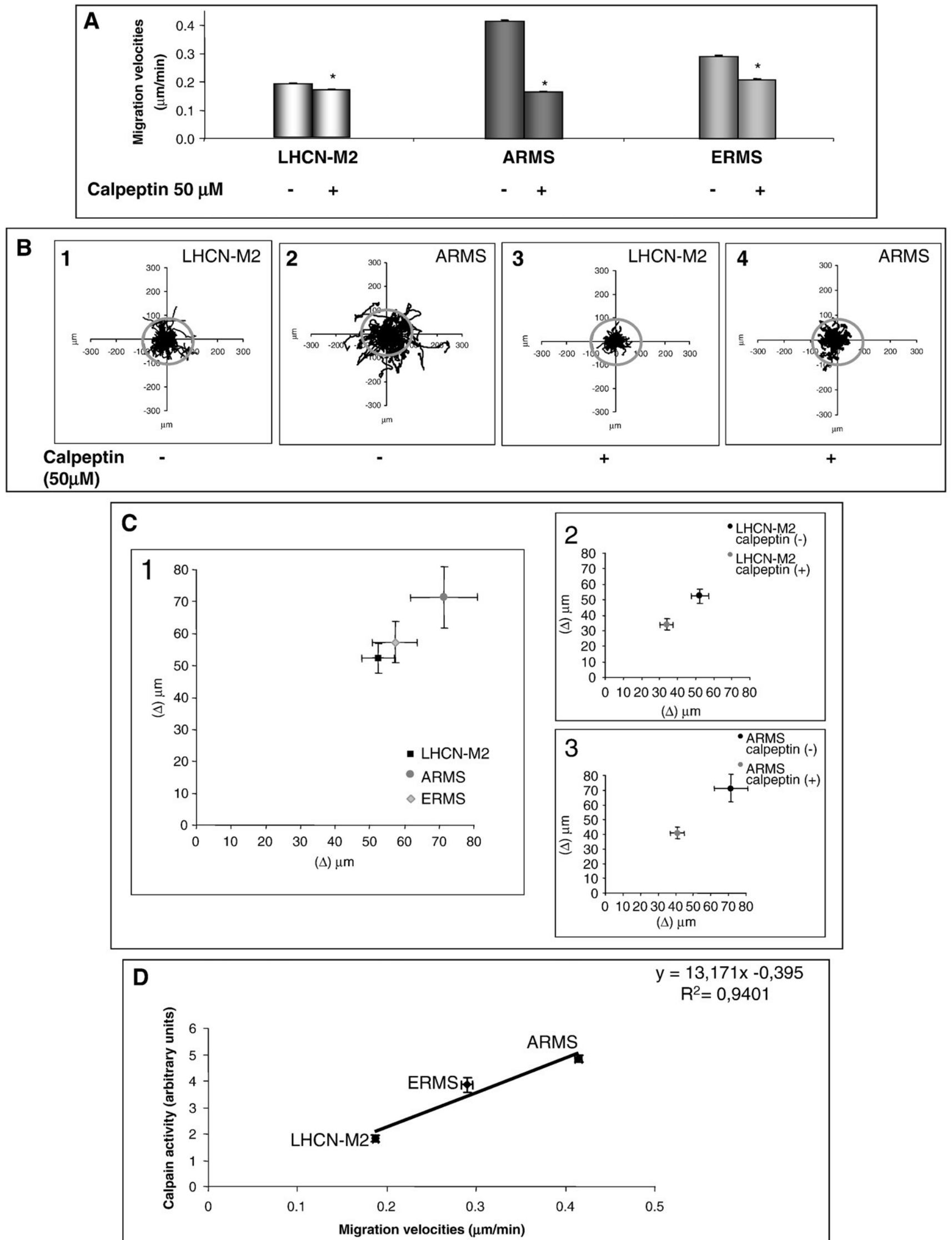

$13,171 x-0,395$

$R^{2}=0,9401$

Migration velocities $(\mu \mathrm{m} / \mathrm{min})$ 

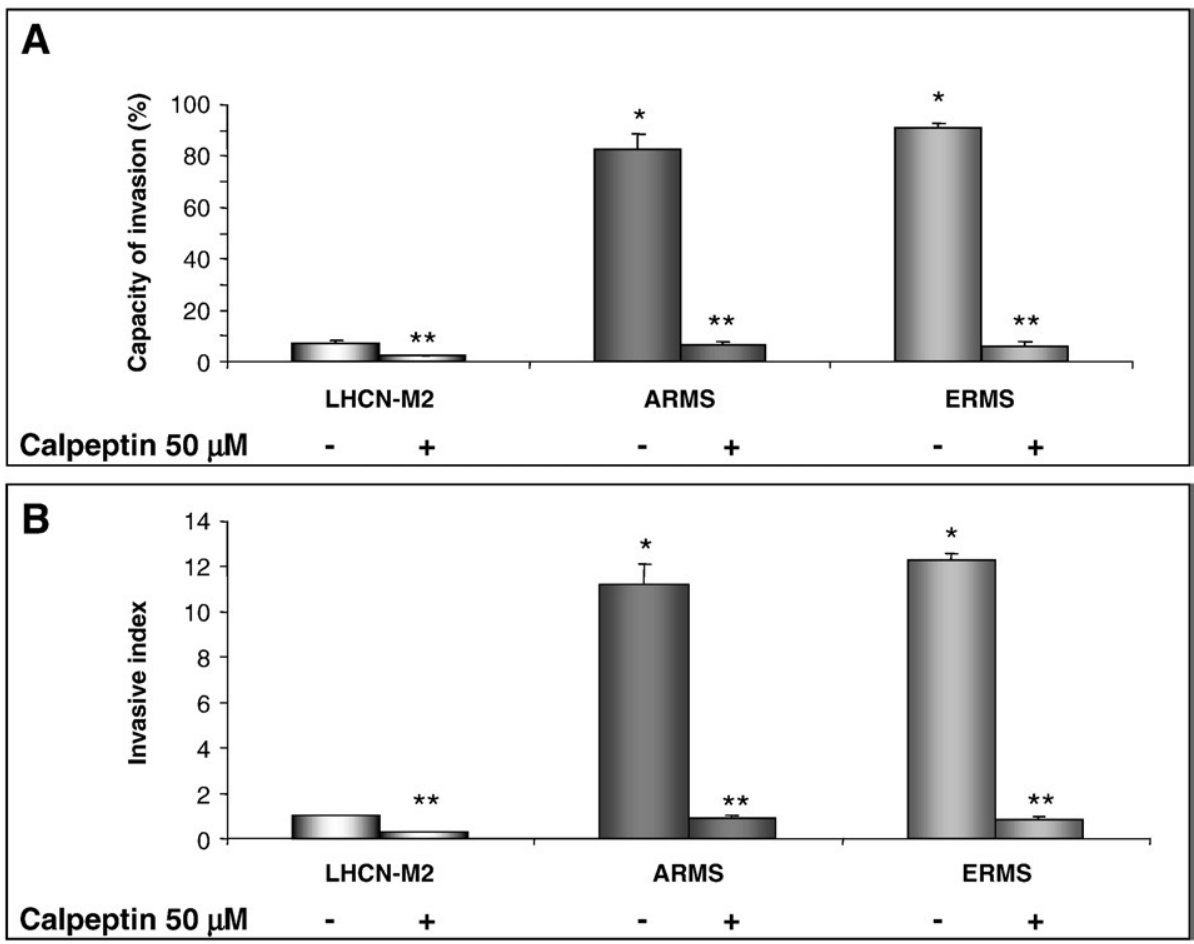

Fig. 6 - Invasive behavior of rhabdomyosarcoma cells. Invasive capacity of LHCN-M2, ARMS and ERMS cells with or without calpeptin $(50 \mu \mathrm{M})(\mathrm{A})$. Invasive index of LHCN-M2, ARMS and ERMS cells with or without calpeptin $(50 \mu \mathrm{M})(\mathrm{B})$. Results are mean values \pm SEM from at least 3 independent experiments. *Significantly different from the control LHCN-M2 cells; **significantly different from data without calpeptin $(\boldsymbol{p}<\mathbf{0 . 0 5})$.

constituting the stress fibers colocalized with $\mu$-calpain. The same colocalization was previously reported in dendritic cells [28] and may implicate $\mu$-calpain in the organization and rearrangement of the actin cytoskeleton. Even if $\mu$-calpain seems to play a role at the time of cell adhesion [29], its association with the stress fibers presumes of a singular role in migration. On the contrary, the actin cytoskeleton of RMS was disorganized and concentrated at the periphery of the cell. This lack of stress fibers has already been described in v-Src transformed cells in which calpain activity simulates the loss of actin stress fibers and induces some morphological modifications [30]. This disorganization may be responsible for the weak adhesion rate and the high migration velocities of RMS cells [31]. In all cell lines, the inhibition of calpain activity induced morphological modifications: cells became rounded, with small protrusions. These changes contrast with the modifications observed in $\mathrm{CHO}$ cells treated with calpain inhibitor 1 which present longer shape than controls [32]. Indeed, fibroblasts over-expressing calpastatin show a rounded morphology as observed in treated LHCN-M2 and RMS cells [33]. In LHCNM2 cells, calpain inhibition induced a disorganization of the central actin cytoskeleton and the F-actin was concentrated at the periphery of the cell. These results are in agreement with those obtained in C2C12 cells over-expressing calpastatin [34]. This rearrangement of actin cytoskeleton was coupled with the relocalization of $\mu$-calpain. This result is in agreement with the changes in $\mu$-calpain localization coordinated with the actinrelated cytoskeleton rearrangements observed during early embryonic development of Drosophila [35]. In LHCN-M2 cells, the persistence of peripheral actin stress fibers, coupled with the disorganization of central fibers could be explained by studies showing that two different pathways are involved in stress fiber regulation: one regulates peripheral stress fibers, depending on calcium and involving calmodulin while the other implies Rho and Rho-kinase [36]. The same concentration of F-actin at the

Fig. 5 - Kinetic characteristics of rhabdomyosarcoma cell lines. Average of migration velocities of all cell lines, with or without calpeptin (A). Individual trajectories of cell displayed in diagrams drown with the initial point of each trajectory placed at the origin of the plot (B). LHCN-M2 and ARMS cells trajectories in standard conditions (B1 and B2, respectively) and in calpeptin presence (B3 and B4, respectively). Distance $\Delta$ between the initial point of each trajectory and the final localization of cell, after $18 \mathrm{~h}$ of time-lapse (C). $\Delta$ for LHCN-M2, ARMS, and ERMS cells, in standard conditions (C1). $\Delta$ for LHCN-M2 and ARMS cells with or without calpeptin (C2 for LHCN-M2 and C3 for ARMS cells). Positive linear correlation between calpain activity and migration velocity (D). Results are mean values \pm SEM from at least 3 independent experiments. *Significantly different from the control LHCN-M2 cells $(\boldsymbol{p}<\mathbf{0 . 0 5})$. 
periphery of the cell was observed in RMS cell lines. These results may involve calpains in the Rho and Rho-kinase-dependant stress fiber regulation.

Although the F-actin was disorganized when calpain activity was inhibited, its quantity was higher in all treated cell lines than in controls. Such increase has already been observed in dendritic cells [28]. In these cells, the accumulation of vinculin, that binds the Arp 2/3 complex, may stimulate the actin polymerization and then increase F-actin quantity. In LHCN-M2 cells, same interactions may be responsible for F-actin increase since inhibition of calpain induced an increase in vinculin expression. On the contrary, in ARMS cells, the increase in F-actin quantity observed when calpain activity was inhibited may result from another process given the fact that calpain inhibition induced a decrease in vinculin expression.

F-actin is formed by $\alpha$-actin and $\beta$-actin. As a consequence, the increase in F-actin caused by the inhibition of calpain may be due to either a stimulation of the polymerization or to an inhibition of the depolymerization. In LHCN-M2 cells, the inhibition of calpains induced an increase of both actin isoforms. Indeed, the increase in F-actin may be due to a stimulation of actin polymerization. These results are in agreement with those of Calle et al. proposing a vinculin-Arp2/3 stimulation of actin polymerization [28]. On the contrary, in the same conditions in ARMS, both actin isoforms decreased. The increase in F-actin observed in ARMS may result from an inhibition of F-actin depolymerization. Moreover, in LHCN-M2 cells, $\mu$-calpain seemed to play a preponderant role in the down-regulation of $\alpha$-actin expression while $m$-calpain downregulated $\beta$-actin expression. In ARMS cells, both $\mu$-calpain and $\mathrm{m}$ calpain had the same effect on $\alpha$-actin and $\beta$-actin. Both calpains stimulated the expression of the two actin isoforms.

To summarize, regulation of actin cytoskeleton organization and arrangement was dependent on calpain activity and on the specific cell type.

The invasive tests realized with all cell lines showed the higher invasive capacities of RMS compared to that of LHCN-M2 cells. This result confirms the invasive phenotype of RMS.

The inhibition of calpain activity induced a decrease in the invasive capacity of all cell lines. These results showed that calpains play a significant role in the invasive process and are in agreement with those obtained in other different cell types such as cancer prostate cells [6], lung cancer cells [8] or osteoclastoma cells [37]. More specifically, in the presence of calpeptin, the invasive capacities of both RMS cell lines were similar to that of LHCN-M2 cells in standard conditions. This result is the second major point of this study directly involving calpain activity in tumor aggressiveness.

The different implications of calpains in adhesion, migration and invasion which are all key steps of metastatic dissemination indicate that $\mu$-calpain and m-calpain are potential therapeutic targets in the aim of stemming tumor invasion and development of metastases. Moreover, the direct relationship between calpain activity and migration velocities or invasive behavior indicates that calpains can serve as useful markers of tumor aggressiveness.

\section{Acknowledgments}

We thank very much Dr V. Mouly and Dr G. S. Butler-Browne for the gift of LHCN-M2 myoblasts and Dr A. Bonnieu for the gift of
RMS. We also thank Dr M. Godfroy for reading the manuscript and A. Pires-Alves for helping with additional experiments.

The authors appreciate the assistance provided by Dr J.-L. Morel for confocal imagery on confocal microscope Leica SP5.

This work was supported by grants from the Association Française contre les Myopathies (AFM), the Ligue National Contre le Cancer, Comités Aquitaine Charente, and from the Institut National de Recherche Agronomique (INRA-France, PHASE Department).

\section{REFERENCES}

[1] P. Dias, D.M. Parham, D.N. Shapiro, B.L. Webber, P.J. Houghton, Myogenic regulatory protein (MyoD1) expression in childhood solid tumors: diagnostic utility in rhabdomyosarcoma, Am. J. Pathol. 137 (1990) 1283-1291.

[2] D.M. Parham, B. Webber, H. Holt, W.K. Williams, H. Maurer, Immunohistochemical study of childhood rhabdomyosarcomas and related neoplasms. Results of an intergroup rhabdomyosarcoma study project, Cancer 67 (1991) 3072-3080.

[3] W.J. Fredericks, N. Galili, S. Mukhopadhyay, et al., The PAX3-FKHR fusion protein created by the $\mathrm{t}(2 ; 13)$ translocation in alveolar rhabdomyosarcoma is a more potent transcriptional activator than PAX3, Mol. Cell. Biol. 15 (1995) 1522-1535.

[4] H.J. Scrable, D.P. Witte, B.C. Lampkin, et al., Chromosomal localization of the human rhabdomyosarcoma locus by mitotic recombination mapping, Nature 329 (1987) 645-647.

[5] R. Dagher, L. Helman, Rhabdomyosarcoma: an overview, Oncologist 4 (1999) 34-44.

[6] A. Mamoune, J.H. Luo, D.A. Lauffenburger, A. Wells, Calpain-2 as a target for limiting prostate cancer invasion, Cancer Res. 63 (2003) $4632-4640$.

[7] L. Xu, X. Deng, Tobacco-specific nitrosamine 4-(methylnitrosamino)-1-(3-pyridyl)-1-butanone induces phosphorylation of mu- and $\mathrm{m}$-calpain in association with increased secretion, cell migration, and invasion, J. Biol. Chem. 279 (2004) 53683-53690.

[8] L. Xu, X. Deng, Suppression of cancer cell migration and invasion by protein phosphatase $2 \mathrm{~A}$ through dephosphorylation of mu- and m-calpains, J. Biol. Chem. 281 (2006) 35567-35575.

[9] E. Schád, A. Farcas, G. Jékely, P. Tompa, P. Friedrich, A novel human small subunit of calpains, Biochem. J. 362 (2002) 383-388.

[10] A. Wendt, V.F. Thompson, D.E. Goll, Interaction of calpastatin with calpain: a review, Biol. Chem. 385 (2004) 465-472.

[11] P. Raynaud, C. Jayat-Vignoles, M.P. Laforêt, H. Levéziel, V. Amarger, Four promoters direct expression of the calpastatin gene, Arch. Biochem. Biophys. 437 (2005) 69-77.

[12] Y. Otsuka, D.E. Goll, Purification of the $\mathrm{Ca}^{2+}$-dependent proteinase inhibitor from bovine cardiac muscle and its interaction with the millimolar $\mathrm{Ca}^{2+}$-dependent proteinase, J. Biol. Chem. 26 (1987) 5839-5851.

[13] C.H. Zhu, V. Mouly, R.N. Cooper, et al., Cellular senescence in human myoblasts is overcome by human telomerase reverse transcriptase and cyclin-dependent kinase 4 : consequences in aging muscle and therapeutic strategies for muscular dystrophies, Aging Cell. 6 (2007) 515-523.

[14] G. Mazères, L. Leloup, L. Daury, P. Cottin, J.J. Brustis, Myoblast attachment and spreading are regulated by different patterns by ubiquitous calpains, Cell. Motil. Cytoskeleton 63 (2006) 193-207.

[15] N.O. Carragher, B.D. Fonseca, M.C. Frame, Calpain activity is generally elevated during transformation but has oncogene-specific biological functions, Neoplasia 6 (2004) 53-73.

[16] L. Leloup, G. Mazères, L. Daury, P. Cottin, J.J. Brustis, Involvement of calpains in growth factor-mediated migration, Int. J. Biochem. Cell. Biol. 38 (2006) 2049-2063. 
[17] J.J. Brustis, N. Elamrani, D. Balcerzak, et al., Rat myoblast fusion requires exteriorized $\mathrm{m}$-calpain activity, Eur. J. Cell. Biol. 64 (1994) 320-327.

[18] L. Leloup, L. Daury, G. Mazères, P. Cottin, J.J. Brustis, Involvement of the ERK/MAP kinase signaling pathway in milli-calpain activation and myogenic cell migration, Int. J. Biochem. Cell. Biol. 39 (2007) 1177-1189.

[19] A. Lambrechts, M. Van Troys, C. Ampe, The actin cytoskeleton in normal and pathological cell motility, Int. J. Biochem. Cell. Biol. 36 (2004) 1890-1909.

[20] C. Braun, M. Engel, M. Seifert, et al., Expression of calpain I messenger RNA in human renal cell carcinoma: correlation with lymph node metastasis and histological type, Int. J. Cancer 84 (1999) 6-9.

[21] J. Reichrath, C. Welter, T. Mitschele, U. Classen, V. Meineke, W. Tilgen, M. Seifert, Different expression patterns of calpain isozymes 1 and 2 (CAPN1 and 2) in squamous cell carcinomas (SCC) and basal cell carcinomas (BCC) of human skin, J. Pathol. 199 (2003) 509-516.

[22] A. Lakshmikuttyamma, P. Selvakumar, R. Kanthan, S.C. Kanthan, R.K. Sharma, Overexpression of $\mathrm{m}$-calpain in human colorectal adenocarcinomas, Cancer Epidemiol. Biomarkers Prev. 13 (2004) 1604-1609.

[23] K. Samanta, P. Kar, B. Ghosh, T. Chakraborti, S. Chakraborti, Localization of m-calpain and calpastatin and studies of their association in pulmonary smooth muscle endoplasmic reticulum, Biochim. Biophys. Acta 1770 (2007) 1297-2307.

[24] E.W. Howard, S.C. Leung, H.F. Yuen, et al., Decreased adhesiveness, resistance to anoikis and suppression of GRP94 are integral to the survival of circulating tumor cells in prostate cancer, Clin. Exp. Metastasis 25 (2008) 497-508.

[25] J. Kassis, R. Radinsky, A. Wells, Motility is rate-limiting for invasion of bladder carcinoma cell lines, Int. J. Biochem. Cell. Biol. 34 (2002) 762-775.

[26] R.G. Taylor, G.H. Geesink, V.F. Thompson, M. Koohmaraie, D.E. Goll, Is Z-disk degradation responsible for postmortem tenderization? J. Anim. Sci. 73 (1995) 1351-1367.
[27] N. Dourdin, A.K. Bhatt, P. Dutt, et al., Reduced cell migration and disruption of the actin cytoskeleton in calpain-deficient embryonic fibroblasts, J. Biol. Chem. 276 (2001) 48382-48388.

[28] Y. Calle, N.O. Carragher, A.J. Thrasher, G.E. Jones, Inhibition of calpain stabilises podosomes and impairs dendritic cell motility, J. Cell. Sci. 119 (2006) 2375-2385.

[29] A. Glading, D.A. Lauffenburger, A. Wells, Cutting to the chase: calpain proteases in cell motility, Trends Cell. Biol. 12 (2002) $46-54$.

[30] N.O. Carragher, V.J. Fincham, D. Riley, M.C. Frame, Cleavage of focal adhesion kinase by different proteases during SRC-regulated transformation and apoptosis. Distinct roles for calpain and caspases, J. Biol. Chem. 276 (2001) 4270-4275.

[31] B.J. Perrin, K.J. Amann, A. Huttenlocher, Proteolysis of cortactin by calpain regulates membrane protrusion during cell migration, Mol. Biol. Cell. 17 (2006) 239-250.

[32] A. Huttenlocher, S.P. Palecek, Q. Lu, et al., Regulation of cell migration by the calcium-dependent protease calpain, J. Biol. Chem. 272 (1997) 32719-32722.

[33] D.A. Potter, J.S. Tirnauer, R. Janssen, et al., Calpain regulates actin remodelling during cell spreading, J. Cell. Biol. 141 (1998) 647-662.

[34] S. Dedieu, S. Poussard, G. Mazères, F. Grise, E. Dargelos, P. Cottin, J. Brustis, Myoblasts migration is regulated by calpain through its involvement in cell attachment and cytoskeletal organization, Exp. Cell. Res. 292 (2004) 187-200.

[35] Y. Emori, K. Saigo, Calpain localization changes in coordination with actin-related cytoskeletal changes during early embryonic development of Drosophila, J. Biol. Chem. 269 (1994) 25137-25142.

[36] K. Katoh, Y. Kano, S. Ookawara, Rho-kinase dependent organization of stress fibers and focal adhesions in cultured fibroblasts, Genes to Cells 12 (2007) 623-638.

[37] D.G. Fan, J.Y. Dai, J. Tang, M.M. Wu, S.G. Sun, J.L. Jiang, Q.Y. Fan, Silencing of calpain expression reduces the metastatic potential of human osteosarcoma cells, Cell. Biol. Int. 33 (2009) 1263-1267. 